\title{
26 Research Square \\ The study of the pathogenesis of idiopathic central precocious puberty based on gut microbiota
}

\section{Congfu Huang ( 78333755@qq.com )}

Maternity and Children Hospital

\section{Bin Wu}

Maternity and Children Hospital

\section{Wei Yang}

Baoan Central Hospital of Shenzhen

\section{Junru Chen}

longgang maternity and child hospital

\section{Zhenyu Yang}

WeHealthGene institute

\section{Yinhu Li}

City University of Hong Kong

\section{Shufen Chen}

longgang maternity and child hospital

\section{Xiangling Wu}

Longgang Central Hospital of Shenzhen

\section{Liping Wu}

longgang maternity and child hospital

\section{Haiying Liu}

maternity and child hospital of shengzhen

\section{Research Article}

Keywords: Idiopathic central precocious puberty1, gut microbiota2, hyperandrogenemia3, insulin resistance4, polycystic ovary syndrome5

Posted Date: July 22nd, 2021

DOl: https://doi.org/10.21203/rs.3.rs-716354/v1

License: (9) (1) This work is licensed under a Creative Commons Attribution 4.0 International License. Read Full License 


\section{Abstract \\ Object}

To analyze the correlation between gut microbiota (GM) and hyperandrogenemia, insulin resistance, carbohydrate metabolism, and explore whether the pathogenesis of idiopathic central precocious puberty (ICPP) and polycystic ovary syndrome (PCOS) is consistent based on GM.

\section{Methods In this study, we have}

recruited 27 ICPP (ICPP group) and 23 healthy children (healthy group), and collected the blood and fecal samples from the participants. Blood samples were tested for hormones, including the follicle-stimulating hormone, luteinizing hormone, estradiol, prolactin, and testosterone. DNA was extracted from fecal samples, and amplified and sequenced with 16S rDNA V3-V4 region. Finally, we annotated the sequencing results, counted the differences in hormone indicators and GM composition between the two groups, and analyzed the correlation with clinical indicators. At the same time, we reviewed the literature on GM and PCOS.

\section{Results}

Compared with the healthy group, the ICPP group exhibited significantly higher levels of the hormone and other indicators $(P<0.05)$. At the phylum level, the ICPP group showed significantly enriched Proteobacteria than the healthy group ( $4.85 \%$ vs $2.92 \%)$. At the genus level, the abundances of Roseburia and Prevotella were significantly higher in the ICPP group than those in the healthy group $(7.55 \%$ vs $2.01 \%, 3.95 \%$ vs $0.19 \%)$, but Bacteroides were obviously decreased in the ICPP group ( $29.96 \%$ vs $44.91 \%)$. In addition, the potential associations underlying the sex hormonal secretion and the carbohydrate metabolism pathways of GM increased significantly in the ICPP group.

\section{Conclusion}

The alternations of GM in ICPP patients are closely related to carbohydrate metabolism, hyperandrogenism, and insulin resistance, indicating similar pathogenesis with polycystic ovary syndrome.

\section{Introduction}

Precocious puberty refers to girls before the age of 8 and boys before the age of 9 with the second sexual sign. Central precocious puberty (CPP) is defined as the onset of the hypothalamus-pituitary-gonadal axis (HPGA) function, and idiopathic central precocious puberty (ICPP) is the most common. At present, the pathogenesis of ICPP is unknown. The main drug for the treatment of CPP is acetate gonadotropin- 
releasing hormone analogue. It must be intramuscularly injected every 4 weeks. The treatment cost is expensive and the treatment time is long. It is necessary to find another treatment as an alternative or auxiliary treatment. It is well known that CPP is associated with genes that affect the development of HPGA. Genes play an important role in the development and regulation of HPGA, the migration and secretion of embryonic gonadotropin-releasing hormone $(\mathrm{GnRH})$ neurons, and the regulation and role of hypothalamic GnRH. In CPP patients, stimulated by GnRH secretion, luteinizing hormone (LH) and folliclestimulating hormone (FSH) secretion pulse and peak also increased, especially LH, LH /FSH ratio increased, high LH directly acted on thecal cells, increased the activity of intracellular straight-chain lyase, stimulated ovarian follicle cells to synthesize androgen early and hyperfunction, resulting in ovarian derived androgen, and LH also induced ovarian synthesis Insulin like growth factor-1(IGF-1) receptor which can increase its binding amount, induce the proliferation of thecal cells, and promote the synthesis and secretion of ovarian androgen (1). CPP is the result of premature activation of the HPGA which affects pubertal gonadotropin levels and progressive characteristics associated with linear growth acceleration and late bone age.

In recent years, the morbidity of central precocious puberty (CPP) has increased significantly. The morbidity of CPP is 5.66/100 million worldwide per year, and most of which are ICPP (2). Studies have reported that patients with precocious puberty may have higher risks of PCOS in adulthood (3), suggesting $t$ the correlations between the two diseases. In addition, abnormal gonadotropin secretion exists in patients with either of the diseases. The cause of PCOS in girls with precocious puberty is not clear, but most of them have hyperinsulinemia, increased early insulin response to blood glucose, increased glucose uptake rate of peripheral tissues, high free androgen index, decreased levels of sex hormone binding globulin (SHBG), and insulin-like growth factor receptor binding protein-1 (IGFBP-1), which are closely related to the characteristics of PCOS $(4,5)$. The occurrence of precocious puberty is closely related to nutrition intake (6-8). Previous study suggested that the intake of excessive high calorie and high fat foods increase the risk of sexual precocity. Children should be encouraged to develop balanced eating habits and increase the intake of high protein foods such as beans and fish. Interesting, diet is closely related to GM, and GM also plays a crucial role in food digestion, nutrition absorption, nutrition metabolism, immune function activation, intestinal barrier and body behavior regulation, which is recognized as an important "microbial organ" of the human body (9). The alterations of GM in PCOS were related to insulin resistance, dyslipidemia and obesity (10). Since the bacteria enriched in the ICPP girls were related to the production of short chain fatty acids (SCFAs), obesity, and sex hormones (11), we wondered whether these two diseases have similar pathogenesis? And how GM affect the occurrence of ICPP? In this study, we recruited 27 ICPP children and 23 healthy age-matched individuals, detected their GM compositions, and analyzed the correlations between the GM compositions, GM functions, and the clinical indicators, such as endocrine hormones.

\section{Materials And Methods}

Ethics Statement 
The study was approved by the Longgang District Maternity \& Child Healthcare Hospital Ethics under Committee number: (2019)LGFYYXLL-024. All procedures were performed following relevant guidelines and regulations stipulated by the Ethics Committee. Before the study, all the parents of the children filled in the written informed consent form, and the parents agreed their children participate in the study.

\section{Research object}

From July 1 to December 31 in 2019, 27 children with ICPP diagnosed in the child health care department of Longgang District Maternity \& Child Healthcare Hospital were selected as the disease group, referred to as ICPP group; another 23 healthy children of the same age who underwent physical examination in the child health care department were recruited as the healthy group. The children in both groups were girls, aged between 6 and 10 years old. The ICPP diagnosis and inclusion criteria as following (12): all patients had a secondary sexual sign before 8 years old, or menarche before 10 years old, ovarian volume $>1 \mathrm{ml}$, multiple follicles with diameter $>4 \mathrm{~mm}, \mathrm{GnRH}$ stimulation test $\mathrm{LH}>5 \mathrm{IU} / \mathrm{L}, \mathrm{LH} / \mathrm{FSH}>0.6$ (chemiluminescence method), CT and MRI excluded central tumor and injury, as well as other organic diseases. All the children did not use antibiotics and had no gastrointestinal symptoms such as diarrhea two weeks before fecal collection.

\section{Clinical observation index}

The clinical data of all enrolled children were investigated by a specially assigned person. The height and weight were measured, and the body mass index (BMI) was calculated: weight ( $\mathrm{kg}) \div$ height $^{2}$ (metre). Blood samples were collected and sent to Guangzhou Jinyu Inspection Center, testing of FSH, LH, fasting insulin, fasting blood glucose and androstenedione(A2) by chemiluminescence method; IGF-1 was detected by tandem mass spectrometry, and Insulin resistance index(HOMA-IR) was calculated : fasting insulin $\times$ Fasting blood glucose / 22.5 .

Fecal sample collection and DNA sequencing

Fecal samples from the ICPP and the healthy children group were collected at the same period of date. About $5 \mathrm{~g}$ fecal were sampled from the middle part of feces. The fecal samples were frozen immediately after collection, stored in $-80^{\circ} \mathrm{C}$, and transported to Beijing novogene Technology Co., Ltd. for 16S rDNA sequencing. All the bacterial DNA was extracted from fecal samples using the PowerSoil@ DNA Isolation Kit. The V3-V4 region of 16S rRNA gene was amplified by PCR, and the amplified sequences were sequenced using Illumina Miseq.

Data analysis

The 16S rDNA sequencing data were filtered by self-designed bioinformatics tools, and Flash software (v1.2.11, http://ccb.jhu.edu/software/FLASH/index.shtml) was used to connect the paired-end reads. Then the connected tags were clustered into OTUs (Operational Taxonomic Units) by usearch. The OTUs were annotated with the Greengene database (V201305), and their relative abundances were calculated 
for all samples. The differentially enriched bacteria between the ICPP and healthy groups were analyzed at the phylum, class, order, family, and genus levels.

Statistical methods

The ADE4 software package of R (v3.3.3) was used to analyze the compositions and relative abundances of genus in all samples. Principal components analysis (PCA) was carried out based on the profiling results, and the overall microbiota distribution of the ICPP and healthy groups was exhibited. SPSS 23.0 was used for statistical analysis. Age, weight, height, and endocrine hormone values of the two groups were compared by two independent sample t-test, and the chi-square test was used for sweet food and high fat in the diet survey. $P<0.05$ was considered to be statistically significant.

\section{Results}

Clinical indicators

Under the same averaged age, the weight, height, BMI, sweet food, and high-fat diet of children in the ICPP group were significantly higher than those in the healthy group $(P<0.05$, Table 1$)$. The levels of serum FSH, LH, estradiol, serum insulin, IGF-1, A2, fasting blood glucose, and HOMA-IR were significantly higher in the ICPP group than those in the healthy children $(P<0.05$, Table 1$)$.

The difference of GM in ICPP girls

\subsubsection{Analysis of dominant bacteria and their difference at the phylum level}

We analyzed the GM compositions in 50 samples from the ICPP and healthy groups (Table 2). At the phylum level, Firmicutes accounted for $52.75 \%$, Bacteroides $38.54 \%$, Proteobacteria $4.85 \%$, Actinobacteria $2.88 \%$, Fusobacteria $0.18 \%$ in the ICPP group. Meanwhile, Firmicutes accounted for $45.81 \%$, Bacteroides 46.95\%, Proteobacteria $2.92 \%$, Actinobacteria $2.59 \%$, Fusobacteria $1.65 \%$ in the healthy group. There were statistically significant differences in Proteobacteria and Fusobacteria between the two groups $(\mathrm{P}<0.05)$.

\subsubsection{Detection of the dominant genera and the differentially enriched genera}

At the genus level, we selected the top 18 dominant genera from each group and counted their proportions respectively (Table 3 ). In the ICPP group, Bacteroides accounted for $29.96 \%$, Roseburia accounted for $7.55 \%$, Prevotella accounted for $3.95 \%$. Whilst, in the healthy group, Bacteroides accounted for $44.91 \%$, Megamonas accounted for $5.25 \%$, and Roseburia accounted for $2.01 \%$. There were many differentially enriched genera existed between the two groups, and we listed the top 18 abundant genera. The dominant genera with significant differences between the two groups included Bacteroides, Roseburia and Prevotella(Table 3). 
Table 3

The top 18 dominant bacteria at genus level in ICPP and healthy groups

\begin{tabular}{|lllll|}
\hline The top 5 phyla & ICPP $(\mathbf{N}=27)$ & Healthy $(\mathbf{N}=23)$ & p-value & FDR-value \\
\hline Firmicutes & $52.75 \pm 11.61$ & $45.81 \pm 18.27$ & 0.1348 & 0.2023 \\
\hline Bacteroidetes & $38.54 \pm 12.92$ & $46.95 \pm 19.22$ & 0.1024 & 0.1843 \\
\hline Proteobacteria & $4.85 \pm 2.58$ & $2.92 \pm 1.86$ & 0.0031 & 0.0094 \\
\hline Actinobacteria & $2.88 \pm 1.78$ & $2.59 \pm 3.03$ & 0.0731 & 0.1646 \\
\hline $0.18 \pm 0.33$ & $1.65 \pm 4.77$ & 0.3003 & 0.3378 & \\
\hline (difference screening conditions: average relative abundance $>0.1 \%, P<0.05, F D R<0.05)$ \\
\hline
\end{tabular}

PCA analysis on the GM at the genus level

The GM similarities between the samples from the two groups were analyzed by PCA. According to the distributions of samples, children from the ICPP group were clustered together, and clear boundaries existed between the two groups (Fig. 1).

Analysis of GM, signal transduction and nutrition metabolism

Due to the GM differences, the functional distributions of GM also different between the ICPP and healthy groups, including the fructose synthesis and metabolism, genetic information processing, nucleotide metabolism, cell process, and signal transmission, energy metabolism, and carbohydrate metabolism. Particularly, the GM in the ICPP group contained the enhanced carbohydrate metabolism (Fig. 2).

The associations between GM and hormones

Performing the correlation analysis between the GM and the clinical hormone levels in the ICPP children, we discovered that the bacteria with higher abundance (e.g. Roseburia, Prevotella, Clostridridium sensu stricto) were significantly associated with the levels of FSH, E2 and testosterone A2, while Sutterella was significantly associated with the above hormone levels in healthy group (Fig. 3).

\section{Discussion}

The results showed that the height and weight of children in the ICPP group increased significantly compared with healthy children of the same age, indicating that children in the ICPP group developed earlier. The analysis of GM with ICPP group prompt, the abundances of Roseburia, Prevotella, Lachnospiracea incertae sedis, Ruminococcus, Parabacteroides, Alistipes, Fuscatinibacter, Clostridium sensu stricto, and Clostridium XVIII were significantly higher than that of the healthy group. Combined with literature analysis, we know that Roseburia belongs to actinomycetes, is one of the most abundant bacteria producing butyric acid in the gut, can promote butyric acid production by decomposing a variety of carbohydrates into SCFAs $(13,14)$. Prevotella can decompose carbohydrates 
into SCFAs and participate in the production of butyrate, which helps to maintain the homeostasis of the gut microenvironment $(14,15)$. Both Ruminococcus and Lachnospiracea incertae sedis, are the two most abundant families of Clostridium in the phylum Firmicutes, they can decompose a variety of fiber polysaccharides. Metabolites of SCFAs are beneficial to the gut, can also promote protein synthesis (16). Clostridium scindens can produce testosterone (17). Fusobacterium was positively correlated with FSH (18). The abundance of Alistipes is closely related to the frequency of abdominal pain, and it is speculated that it may lead to gut inflammation (19). The increase of the abundance of Parabacteroides can cause the reduction of the synthesis of tight junction protein (tight junction protein-1 and atresia protein), thus leading to the occurrence of "gut leakage" (20). These results suggest that the change of GM in the ICPP group can lead to the increase of SCFAs production, especially butyrate content, and the increase of some microbiota abundance can lead to chronic gut inflammation. Some studies $(21,22)$ believe that butyrate can directly stimulate the secretion of glucagon-like peptide-1 (GLP-1) by endocrine cells gpr43 or gpr41 in colon and ileum, and the latter can promote insulin secretion. Also found that butyrate can stimulate the increase of nitric oxide (NO) synthesis when the concentration of butyrate is significantly increased (23).

This study confirmed the existence of intestinal dysbacteriosis in children with ICPP. We further analyzed the function of GM and analyzed the correlation between GM and endocrine hormones with the ICPP group. Hence, we speculate that the changes of GM with ICPP and PCOS were related to endocrine hormone disorders in the two diseases. PCOS patients have high calorie and high-fat diet, insulin resistance, hyperandrogenemia, and so on. Combined with our research results, we analyzed ICPP children from these three aspects.

\subsection{Dietary structure}

Previous investigation (7) suggested that precocious puberty children preferred high-fat and high-calorie diets. This study also found that children in the ICPP group preferred sweet food (high sugar diet) and high-fat food, we know that the high sugar diet belonged to low molecular carbohydrate. Moreover, we found the abundance of bacteria such as Prevotella that carbohydrate metabolizing in GM was significantly increased, and carbohydrate metabolism in GM was significantly enhanced with the ICPP group, so we speculate that a high sugar diet may be an important factor in precocious puberty.

It has been reported (24) that high fat and high sugar diet can induce metabolic disorder and ovarian changes in female rats. Similar clinical manifestations to PCOS patients have been observed. It has been confirmed that low calorie and low-fat diet is an effective therapy to improve metabolism and ovarian function in obese women with PCOS (25).

\subsection{Hyperandrogenemia}


The negative feedback of hyperandrogenemia caused the decrease of estradiol and progesterone secretion and the increase of GnRH and LH levels (26). The synthesis of androgen in women is closely related to HPGA. The former mainly comes from the androgen secreted by the ovarian tissue. The testosterone and A2 secreted by the ovary account for two-thirds of the circulating androgens (27). The androgen synthesis in the ovary is regulated by $\mathrm{LH}$. Total serum testosterone is the most common and widely used biochemical test index to evaluate androgen excess (28). However, there are many defects in the detection method of testosterone in clinical practice. At present, androgen level is evaluated by A2 in PCOS patients (29). Wang Quanxian et al. (30) believe that A2 expression is closely related to endocrine and metabolic disorders in PCOS patients. In this project, A2 in the ICPP group was significantly higher than that in healthy group, which may be caused by the following factors: 1) According to the investigation, the children in the ICPP group like to eat sweet food (carbohydrate intake is more), and the correlation analysis between GM and hormone level of children also indicates that the metabolism of carbohydrate in children in the ICPP group is significantly higher than that in the healthy group, and high sugar diet can cause an immune response and oxidative stress to produce inflammatory mediators and stimulate ovaries to produce androgen (31). 2) Compared with healthy children, the bacterial abundance of butyric acid-producing bacteria in the ICPP group was significantly increased, butyric acid-induced insulin secretion through GLP-1 $(26,32)$, insulin enhanced GnRH gene transcription by enhancing mitogen-activated protein kinase pathway, and increased $\mathrm{GnRH}$ secretion in the hypothalamus, resulting in high levels of androgen and LH secretion. On the other hand, insulin can improve the utilization of IGF1 by inhibiting the expression of IGFBP-1 in the ovary and liver. Insulin can also improve IGF-1 activity in the liver and ovary, reduce the level of SHBG in the liver and ovary, and increase free androgen in vivo $(26,33) .3)$ In the ICPP group, the abundance of butyric acid-producing bacteria such as Roseburia, Prevotella, Lachnospiracea incertae sedis, and Ruminococcus increased, which may lead to the rich energy of intestinal mucosal cells, the secretion of adrenocorticotropic hormone(ACTH) and other hormones in the gut tract, and also affect the HPGA to promote the synthesis of androgen (33). 4) The abundance of Clostridium sensu stricto and Clostridium XVIII in the ICPP group is increased, the latter can convert glucocorticoid into androgen, studies have confirmed that the bacterium can produce testosterone $(34,35) .60 \%$ of testosterone in women is converted from its direct precursor A2.

Liu et al. (36) reported that LPS-producing bacteria such as Bacteroides and Escherichia/Shigella increased in PCOS patients. The correlation analysis showed that the above bacteria were positively correlated with androgen level, suggesting that the changes of GM could lead to hyperandrogenism. The female newborn rats were given a large dose of testosterone once, which not only showed similar phenotypes of PCOS, such as weight gain and insulin resistance but also caused the continuous changes of GM. It suggested that androgen was related to GM disorder, the former could promote the development of PCOS (37).

\subsection{Insulin resistance}


Insulin resistance is one of the main pathogenesis of precocious puberty, which can enhance the regulation effect of upstream $\mathrm{LH}$ on the ovary and strengthen the function of the adrenal gland to synthesize androgen; it can also inhibit the synthesis of SHBG in the liver, reduce the binding of androgen and SHBG in serum, and cause the increase of free testosterone level (38). Analysis of the causes of insulin resistance in the ICPP group: on the one hand, the increase of androgen level can cause gut mucosal damage (39), and the abundance of Alistipes and Parabacteroides increased can lead to gut inflammation $(20,40)$. As a result, the permeability of gut mucosa is increased. Many inflammatory mediators produced by GM, such as lipopolysaccharide (LPS) and branched-chain amino acid (BCAA), can activate the immune response. Meanwhile, inflammatory mediators can stimulate the production of various inflammatory factors by activating toll-like receptors 4 (TLR4). At the same time, through nuclear factor - KB (NF-KB) and other signaling pathways, it can promote the phosphorylation of insulin receptor substrate-1 (IRS-1) in the insulin signaling pathway, resulting in the decrease of insulin sensitivity. On the other hand, the increase of butyric acid-producing bacteria can also stimulate the increase of GLP-1 content $(26,32)$, in our study, the levels of IGF-1 and insulin in the ICPP group were significantly higher than those in the same age healthy group, which also supported the above conclusion. In addition, the insulin resistance index of ICPP children was significantly increased in this study, we analyzed the reasons for the existence of insulin resistance: first, LPS produced by GM can induce chronic inflammation in the intestine, and lead to insulin resistance by activating TLR4 (38). Second, our previous study also found that (41) ICPP children have increased NO synthesis in GM, which is positively correlated with FSH and insulin levels, and NO can promote insulin resistance. Last, Prevotella abundance was increased in ICPP children in our study, and Prevotella could increase circulating BCAA level, the latter was positively correlated with free testosterone level and insulin resistance $(42,43)$.

Liu et al. (36) analysis of GM in PCOS patients showed that the abundance of LPS-producing bacteria such as Bacteroides and Escherichia/Shigella increased, and the abundance of intestinal barrier protection bacteria such as Akkermensia decreased, which led to the increase of LPS production. Compared with the serum LPS binding protein (LBP) in PCOS and healthy people, Zhu et al. (44) found that the level of LBP in PCOS patients was significantly increased, and the combination of LPS and LBP could cause the expression of tumor necrosis factor- $a$ (TNF- $a$ ) and IL-6, which is related to insulin resistance (45). Roychoudhury et al. (46) also found that the blood valine and leucine content in PCOS patients increased, 3-hydroxybutyrate (3-HIB), the metabolite of valine, stimulated the intake of fatty acids in muscle tissue, which could cause fat accumulation and insulin resistance (47).

\subsection{Summary and thinking}

Barrea et al. (48) considered that the interaction between high carbohydrate intake, hyperinsulinemia, hyperandrogenemia, and chronic low-grade inflammation are the four key factors for pathophysiological changes of PCOS. The correlation between hyperandrogenemia, insulin resistance, and chronic low inflammation in patients with ICPP and PCOS was analyzed. In this project, the children in the ICPP group liked to eat sweet food (which may lead to excessive carbohydrate intake), and increased carbohydrate 
metabolism in GM, so we think that ICPP and PCOS have similar pathogenesis. However, there are also differences between ICPP and PCOS. The latter has a low estrogen level, and the estrogen level of the ICPP group is higher than that of the healthy group. Based on the above research and analysis, we believe that the changes of GM in ICPP children will affect the changes of intestinal short-chain fatty acid composition, thus promoting the secretion of sex hormones such as androstenedione, IGF-1, and insulin. The structural changes of GM will lead to chronic low-grade inflammation, hyperandrogenemia, and insulin resistance, and finally start the HPGA in advance. These factors can also activate the GH-IGF-1 axis, promote growth hormone secretion through IGF-1, and lead to the sudden increase in height and weight, which may be the pathogenesis of ICPP children. The sample size of this study is small, and the fecal samples are only for $16 \mathrm{~S}$ rRNA sequencing. The next step is to carry out the large sample and multicenter research. It is better to take macro genre sequencing for fecal samples, combined with the analysis of GM metabolites and blood metabolites, which may be more helpful to clarify the pathogenesis of ICPP.

\section{Declarations}

\section{Funding}

This work has been strongly supported by Longgang District Science and technology innovation Bureau (LGKCYLWS2020104).

\section{Acknowledgements}

We thank all participants for their support. We thank the doctors and nurses of Longgang District Maternity \& Child Healthcare Hospital (Shenzhen, China) and Shenzhen Ninth People's Hospital (Shenzhen, China) for assisting the research team in clinical examination and fecal sample collection. We also thank the authors who made their data publicly available.

\section{Ethics statement}

This study was approved by the ethics committee of Longgang District Maternity \& Child Healthcare Hospital of Shenzhen city with the registration number of LGFYYXLL-024. The parents, as legal guardians, voluntarily accepted scientific research on their children's care.

\section{Conflict of interest}

The authors declare that the research was conducted in the absence of any commercial or financial relationships that could be construed as a potential conflict of interest. 


\section{Author contributions}

Huang Congfu, Wu Bin and Liu Haiying manage the project. Wu Bin, Chen Junru, Chen Shufen and Wu Xiangling collected fecal samples and information. Huang Congfu, Liu Haiying and Yang Zhenyu prepared the DNA. Yang Zhenyu and Li Yinhu are responsible for bioinformatics analysis in this work. Huang Congfu, Wu Bin and Xiao Zhengxiang explained the analysis results and wrote a paper. Yang Zhenyu and Wu Liping optimized the graph and statistical analysis. Xiao Zhengxiang guides the design and embellishment of articles. All the authors reviewed the manuscript.

\section{Data availability statement}

The data set generated for this study can be read from the NCBI sequence Archive (SRA) database, biological project number: (prjna67248). This study was registered in China clinical trial center, registration number: ChiCTR2000033305.

\section{References}

1. Wu CF, Liu Y (2011) Research Progress on Mechanism of Ovariogenic Hyperandrogenism of Polycystic Ovary Syndrome. Medicine Healthcare Apparatus 18(1):147-149. https://doi.org/10.3969/j.issn.1674-4659.2011.01.0147

2. Soriano-Guillén L, Corripio R, Ignacio Labarta J, Cañete R, Castro-Feijóo L, Espino R et al (2010) Central precocious puberty in children living in Spain:incidence,prevalence,and influence of adoption and immigration. J Clin Endocrinol Metab 5(9):4305-4313. https://doi.org/10.1210/jc.2010-1025

3. Peng W, Yang FQ (2012) Research Progress on the relationship between precocious puberty and polycystic ovary syndrome. Anhui Medical Journal 33(5):635-637.

.https://doi.org/10.3969/j.issn.1000-0399.2012.05.050

4. Su Z, Ma HM, Li YH, Du ML, Chen HS, Liu M,et al (2008) Change of serum dehydroepiandrosterone sulfate (DHEAS) level in children and adolescents. Chinese Journal of Laboratory Medicine 31(12):1358. https://doi.org/10.3321/j.issn:1009-9158.2008.12.009

5. Zhang YH, Yan ML, Zhang D (2017) Discussion of relationships between hormone, IGF-1 and growth and development in young girls with central precocious puberty. China Medical Herald 14(35):75-78

6. Ullah R, Su Y,Shen Y, Li CL,Xu XQ, Zhang JW,et al (2017) Postnatal feeding with high-fat diet induces obesity and precocious puberty in C57BL/6J mouse pups:a novel model of obesity and puberty. Front Med 11(2):266-276. https://doi.org/10.1007/s11684-017-0530-y

7. Meng FS, Chen DY, Wu Y, Su Z,Xie HW,Zhou L (2020) Study of relationship between dietary patterns and precocious puberty of school-age girls in Shenzhen. Chinese Journal of Epidemiology 41(5):738-742. https://doi.org/10.3760/cma.j.cn112338-20190630-00478 
8. Chen C, Chen Y, Zhang Y, Sun WQ, Jiang YR, Song YJ,et al (2018)Association between Dietary Patterns and Precocious Puberty in Children: A Population-Based Study. Int J Endocrinol 4528704. https://doi.org/10.1155/2018/4528704

9. Lopategi A, López-Vicario C, Alcaraz-Quiles J, García-Alonso V, Rius B, Titos E,et al (2016) Role of bioactive lipid mediators in obese adipose tissue inflammation and endocrine dysfunction. Mol Cell Endocrinol 419:44-59. https://doi.org/10.1016/j.mce.2015.09.033

10. Liu HP, Du H, Li N, Gao SJ, Liu GZ (2019)Role of intestinal flora imbalance in metabolic abnormalities of polycystic ovary syndrome.Journal of Practical Obstetrics and Gynecology 35(4):263265.https://doi.org/CNKI:SUN:SFCZ.0.2019-04-010.

11. Dong GQ, Zhang JY, Yang ZY, Feng X, Li JX, Li DF,et al (2020) )The Association of Gut Microbiota With Idiopathic Central Precocious Puberty in Girls. Front Endocrinol (Lausanne) 1(10):941. https://doi.org/10.3389/fendo.2019.00941

12. Department of Endocrinology, genetics and metabolism, Chinese Medical Association (2007) Guidelines for diagnosis and treatment of central precocious puberty. Chin J Pediatr 45(6):426

13. Travis AJ, Kelly D, Flint HJ, Aminov RI (2015) Complete Genome Sequence of the Human Gut Symbiont Roseburia hominis. Genome Announc 3(6):e01286-e01215. https://doi.org/10.1128/genomeA.01286-15

14. Zhang JD, Song LJ,Wang YJ,Liu C, Zhang L, Zhu SW,et al (2019) Benefificial effect of butyrateproducing Lachnospiraceae on stress-induced visceral hypersensitivity in rats. J Gastroenterol Hepatol 34(8):1368-1376. https://doi.org/10.1111/jgh.14536

15. Tremlett H, Bauer KC, Appel-Cresswell S, Finlay BB,Waubant E (2017)The gut microbiome in human neurological disease: A review. Ann Neurol 81(3):369-382.https://doi.org/ 10.1002/ana.24901

16. Chen WG, Liu FL,Ling ZX, Tong XJ,Xiang CL (2012)Human Intestinal Lumen and Mucosa-Associated Microbiota in Patients with Colorectal Cancer. PLo S ONE 7(6): e39743.https://doi.org/ 10.1371/journal.pone.0039743

17. Ridlon JM, Ikegawa S, Alves JMP, Zhou B, Kobayashi A, lida T,et al (2013) Clostridium scindens: a human gut microbe with a high potential to convert glucocorticoids into androgens. J Lipid Res 54(9):2437-2449. https://doi.org/10.1194/jlr.M038869

18. Dong GQ, Zhang JY, Yang ZY, Feng X, Li JX, Li DF,et al (2020) )The Association of Gut Microbiota With Idiopathic Central Precocious Puberty in Girls. Front Endocrinol (Lausanne) 1(10):941. https://doi.org/10.3389/fendo.2019.00941

19. Saulnier DM, Riehle K, Mistretta TA, Diaz MA, Mandal D, Raza S,et al (2011) Gastrointestinal microbiome signatures of pediatric patients with irritable bowel syndrome. Gastroenterology 141(5):1782-1791. https://doi.org/10.1053/j.gastro.2011.06.072

20. Lee SM, Han HW,Yim SY (2015) Beneficial effects of soy milk and fiber on high cholesterol dietinduced alteration of gut microbiota and inflammatory gene expression in rats. Food function 6(2):492-500. doi:10.1039/c4fo00731j 
21. Burokas A, Moloney RD, Dinan TG, Cryan JF (2015) Microbiota Regulation of the Mammalian Gutbrain Axis. Adv Appl Microbiol 91:1-62. https://doi.org/10.1016/bs.aambs.2015.02.001

22. Tolhurst G, Heffron H, Yu SL, Parker HE,Habib AM,Diakogiannaki E,et al (2012) Short-chain fatty acids stimulate glueagon-like peptide-1 secretion via the G-protein-coupled receptor FFAR2. Diabetes 61(2):364-371. https://doi.org/10.2337/db11-1019

23. Hu S, Kuwabara R, de Haan BJ,et al (2020) Acetate and Butyratelmprove beta-cell Metabolism and Mitochondrial Respiration under Oxidative Stress. Int J Mol Sci 21(4):1542. https://doi.org/10.3390/ijms21041542

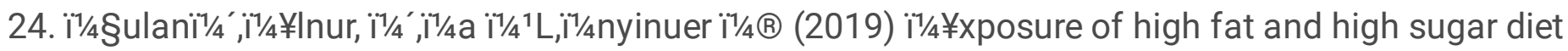

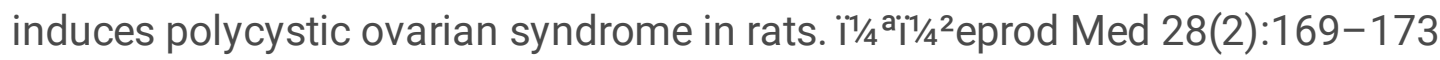

25. Azadi-Yazdi M, Karimi-Zarchi M, Salehi-Abargouei A, Fallahzadeh H, Nadjarzadeh A (2017) )Effects of Dietary Approach to Stop Hypertension diet on androgens, antioxidant status and body composition in overweight and obese women with polycystic ovary syndrome: a randomised controlled trial. J Hum Nutr Diet 30(3):275-283. https://doi.org/10.1111/jhn.12433

26. Rojas J, Chdvez M, Olivar L, Rojas M,Morillo J,Mejías J,et a1 (2014)Polycystic ovary syndrome,insulin resistance,and obesity:navigating the pathophysiologic labyrinth. Int J Reprod Med 2014:719050.https://doi.org/10.1155/2014/719050

27. Azziz R, Carmina E, Dewailly D, Diamanti-Kandarakis E, Escobar-Morreale HF, Futterweit W,et al (2009) The Androgen Excess and PCOS Society criteria for the polycystic ovary syndrome:the complete task force report. Fertil Steril 91(2):456-488. https://doi.org/10.1016/j.fertnstert.2008.06.035

28. Brian G,Keevil (2014)How Do We Measure Hyperandrogenemia in Patients With PCOS? J Clin Endocrinol Metab 99(3):777-779.https://doi.org/10.1210/jc.2014-1307

29. Xu ZQ, Wang XL (2016)Diagnostic accuracy of androstenedione in polycystic ovary syndrome with hyperandrogenemia. Master's thesis 1-35.https://doi.org/10.7666/d.D834488

30. Wang QX, Liu JJ, sun XT, Song ZZ (2018) Relationship between serum androstendione level of patients with PCOS and its metabolic characteristics. Journal Of Tropical Medicine 18(3):371376.https://doi.org/CNKI:SUN:RDYZ.0.2018-03-022.

31. Gonzalez F, Rote NS,Minium J, Kirwan JP (2006) In vitro evidence that hyperglycemia stimulates tumor necrosis factor-a release in obese women with polycystic ovary syndrome. J Endocrinol 188(3):52l-529. https://doi.org/10.1677/joe.1.06579

32. Khan S, Jena G (2016) Sodium butyrate reduces insulin-resistance, fat accumulation and dyslipidemia in type-2 diabetic rat:A comparative study with metformin. Chem Biol Interact 254:124134. https://doi.org/10.1016/j.cbi.2016.06.007

33. Tan J, Mckenzie C,Potamitis M, Thorburn AN,Mackay CR,Macia L (2014) The role of short-chain fatty acids in health and disease. Adv Immunol 121:91-119. https://doi.org/10.1016/B978-0-12-8001004.00003-9 
34. Ridlon JM, Ikegawa S, Alves JMP, Zhou B, Kobayashi A, lida T,et al (2013) Clostridium scindens: a human gut microbe with a high potential to convert glucocorticoids into androgens. J Lipid Res 54(9):2437-2449. https://doi.org/10.1194/jlr.M038869

35. Baker JM, Al-Nakkash L, Herbst-Kralovetz MM (2017) Estrogen-gut microbiome axis: physiological and clinical implications. Maturitas 103:45-53.

https://doi.org/10.1016/j

36. maturitas.2017.06.025

37. Liu R, Zhang C, Yu S,ZhangF, Li LX, Wang XJ,et al (2017)Dysbiosis of gut microbiota associated with clinical parameters in polycystic ovary syndrome.Front Microbiol 8 :

324.https://doi.org/10.3389/fmicb.2017.00324. eCollection 2017

38. Moreno-Indias I, Sán-AL,Sán-GM,Martín-Núñez GM, Pérez-Jiménez F, Tena-Sempere M,et al (2016)Neonatal androgen exposure causes persistent gut microbiota dysbiosis related to metabolic disease in adult female rats.Endocrinology 157:4888-4898.https://doi.org/10.1210/en.2016-1317. Epub 2016 Oct 4

39. Ye LX, Hong J (2019) Polycystic ovary syndrome and gut microbiota. International Journal of Endocrinology Metabolism 39(5):345-348

40. Homma H, Hoy E, Xu DZ, Lu Q, Feinman R, Deitch EA (2005)The female intestine is more resistant than the male intestine to gut injury and infflammation when subjected to conditions associated with shock states. Am J Physiol Gastr Liver Physiol 288 (3): G466-G472.https://doi.org/ 10.1152/ajpgi.00036.2004

41. Griffin JL, Wang X, Stanlet E (2015) Does our gut microbiomepredict cardiovascular risk? A review of the evidence frommetabolomics. Circulation Cardiovascular Genetics 8(1):187-191. https://doi.org/10.1161/CIRCGENETICS.114.000219

42. Li YH, Shen L, Huang CF, Li XY, Chen JR, Li SC, Shen BR (2021) Altered nitric oxide induced by gut microbiota reveals the connection between central precocious puberty and obesity. Clin Transl Med 11(2):e299. https://doi.org/10.1002/ctm2.299

43. Chang AY, Lalia AZ,Jenkins GD, Dutta T, Carter RE,Singh RJ,et al (2017)Combining a nontargeted and targeted metabolomics approach to identify metabolic pathways significantly altered in polycystic ovary syndrome.Metabolism 71: 52-63.https://doi.org/10.1016/j.metabol.2017.03.002

44. Pedersen HK, Gudmundsdottir V, Nielsen HB,Hyotylainen T, Nielsen T, Jensen BAH,et al (2016)Human gut microbes impact host serum metabolome and insulin sensitivityNature 535(612):376381.https://doi.org/10.1038/nature18646. Epub 2016 Jul 13

45. Zhu Q, Zhou H, Zhang A, Gao R, Yang SM, Zhao CH,et al (2016) Serum LBP is associated with insulin resistance in women with PCOS. PLoS One 11(1):e0145337.

https://doi.org/10.1371/journal.pone.0145337. eCollection 2016

46. Wang YY, Liu Y, Huang HF (2018)Roles of gut microbiota in the occurrence and development of polycystic ovary syndrome.Journal Of Shanghai Jiao Tong University(Medical Science) 38(4):454457.https://doi.org/10.3969/j.issn.1674-8115.2018.04.019 
47. Jang C, Oh SF, Wada S, Rowe GC, Liu L, Chan MC,et al (2016) A branched-chain amino acid metabolite drives vascular fatty acid transport and causes insulin resistance. Nat Med 22(4):421426. https://doi.org/10.1038/nm.4057

48. RoyChoudhury S, Mishra BP, Khan T, Chattopadhayay R, Lodh I, Ray CD,et al (2016) Serum metabolomics of Indian women with polycystic ovary syndrome using $1 \mathrm{H}$ NMR coupled with a pattern recognition approach. Mol Biosyst 12(11):3407-3416. https://doi.org/10.1039/c6mb00420b

49. Barrea L, Marzullo P, Muscogiuri G, Somma CD,Scacchi M, Orio F,et al (2018) Source and amount of carbohydrate in the diet and inflammation in women with polycystic ovary syndrome. Nutr Res Rev 31(2):291-301. https://doi.org/10.1017/S0954422418000136

\section{Tables 1 And 2}

Table 1. The clinical information of the ICPP and healthy girls

\begin{tabular}{|c|c|c|c|}
\hline Characteristics & ICPP $(N=27)$ & healthy $(N=23)$ & $p / x^{2}$-value \\
\hline Age (mean $\pm S D$, year) & $7.72 \pm 0.45$ & $7.4 \pm 0.77$ & 0.204 \\
\hline Height (mean \pm SD, cm) & $138.89 \pm 6.36$ & $129.87 \pm 6.39$ & 0.000 \\
\hline Weight (mean \pm SD, kg) & $34.92 \pm 6.92$ & $26.31 \pm 5.29$ & 0.000 \\
\hline $\mathrm{BMI}($ mean \pm SD) & $18.05 \pm 2.97$ & $15.64 \pm 3.10$ & 0.008 \\
\hline Number of sweet eaters (n) & $19 / 27$ & $8 / 23$ & 0.012 \\
\hline Number of high fat eaters ( & (n) $17 / 27$ & $8 / 23$ & 0.047 \\
\hline $\mathrm{FSH}($ mean $\pm \mathrm{SD}, \mathrm{mlU} / \mathrm{ml})$ & $4.02 \pm 1.75$ & $2.09 \pm 1.02$ & 0.000 \\
\hline $\mathrm{LH}($ mean $\pm \mathrm{SD}, \mathrm{mlU} / \mathrm{m})$ & $1.39 \pm 1.17$ & $0.17 \pm 0.21$ & 0.000 \\
\hline E2 (mean \pm SD, pmol/L) & $27.44 \pm 18.05$ & $2.61 \pm 8.80$ & 0.001 \\
\hline Insulin (mean $\pm \mathrm{SD}, \mu \mathrm{U} / \mathrm{mL}$ ) & $10.07 \pm 5.52$ & $5.114 \pm 3.78$ & 0.000 \\
\hline A2 (mean $\pm S D, \mu g / L)$ & $2.06 \pm 0.37$ & $0.73 \pm 0.42$ & 0.000 \\
\hline IGF-1 (mean \pm SD, ng/mL) & $308.19 \pm 54.42$ & $205.66 \pm 49.00$ & 0.000 \\
\hline $\mathrm{BS}($ mean $\pm \mathrm{SD}, \mathrm{mmol} / \mathrm{L})$ & $4.85 \pm 0.79$ & $4.35 \pm 0.64$ & 0.018 \\
\hline HOMA-IR (mean \pm SD) & $2.23 \pm 1.38$ & $1.05 \pm 0.89$ & 0.01 \\
\hline
\end{tabular}


FSH, Follicle stimulating hormone; LH, Luteinizing hormone; E2, Estradiol; A2, androstenedione; IGF-1, Insulin-like growth factor 1;BS,blood sugar; HOMA-IR, Insulin resistance index

Table 2. The top 5 dominant bacteria at phylum level in ICPP and healthy groups (difference screening conditions: average relative abundance $>0.1 \%, \mathrm{P}<0.05, \mathrm{FDR}<0.05$ )

\begin{tabular}{|lllll|}
\hline The top 5 phyla & ICPP $(\mathbf{N}=27)$ & Healthy $(\mathbf{N}=23)$ & p-value & FDR-value \\
\hline Firmicutes & $52.75 \pm 11.61$ & $45.81 \pm 18.27$ & 0.1348 & 0.2023 \\
\hline Bacteroidetes & $38.54 \pm 12.92$ & $46.95 \pm 19.22$ & 0.1024 & 0.1843 \\
\hline Proteobacteria & $4.85 \pm 2.58$ & $2.92 \pm 1.86$ & 0.0031 & 0.0094 \\
\hline Actinobacteria & $2.88 \pm 1.78$ & $2.59 \pm 3.03$ & 0.0731 & 0.1646 \\
\hline Fusobacteria & $0.18 \pm 0.33$ & $1.65 \pm 4.77$ & 0.3003 & 0.3378 \\
\hline
\end{tabular}

Figures 


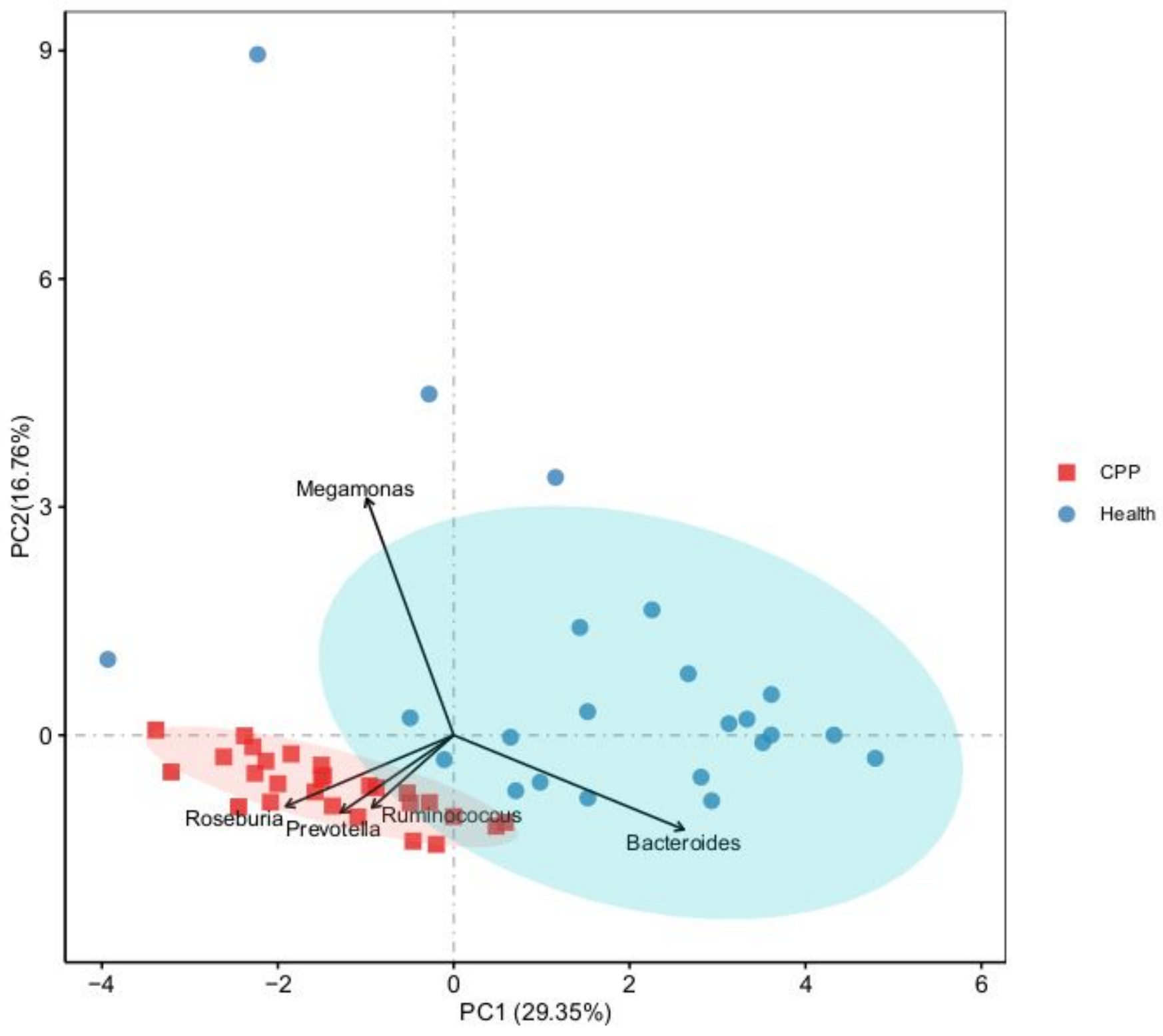

\section{Figure 1}

The two groups of samples were analyzed by principal component analysis, according to the cluster of samples, children in the ICPP group were more clustered, but the healthy group formed an interaction network with Bacteroides, Megamonas, Megasphaera as the center. Therefore, the bacterial network of the two groups was different. 


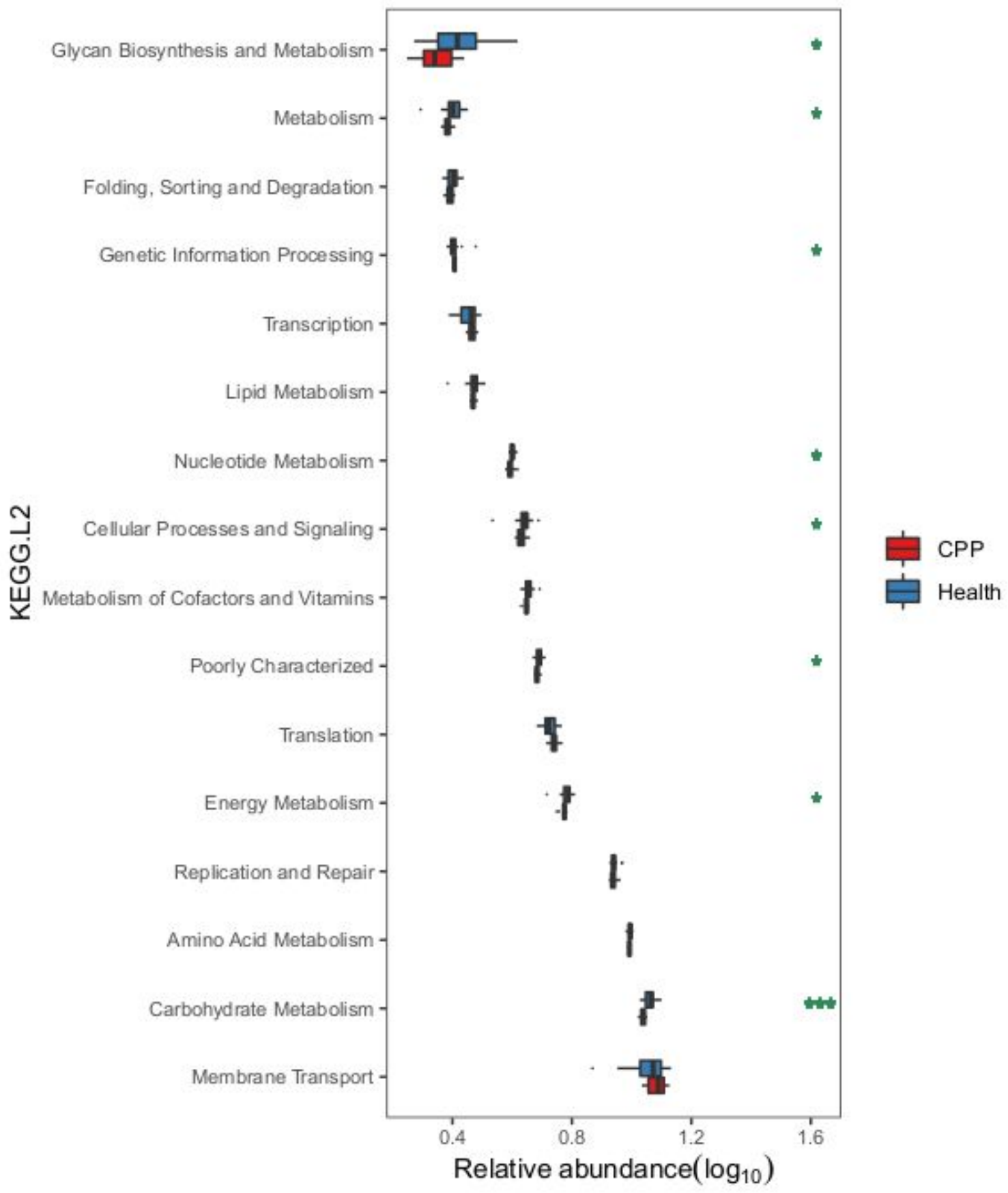

Figure 2

There were differences in fructose synthesis and metabolism, genetic information processing, nucleotide metabolism, cell process, and signal transmission, energy metabolism, and carbohydrate metabolism between the ICPP group and the healthy group $(P<0.05)$. In particular, carbohydrate metabolism was significantly enhanced in the ICPP group $(P<0.001)$. 


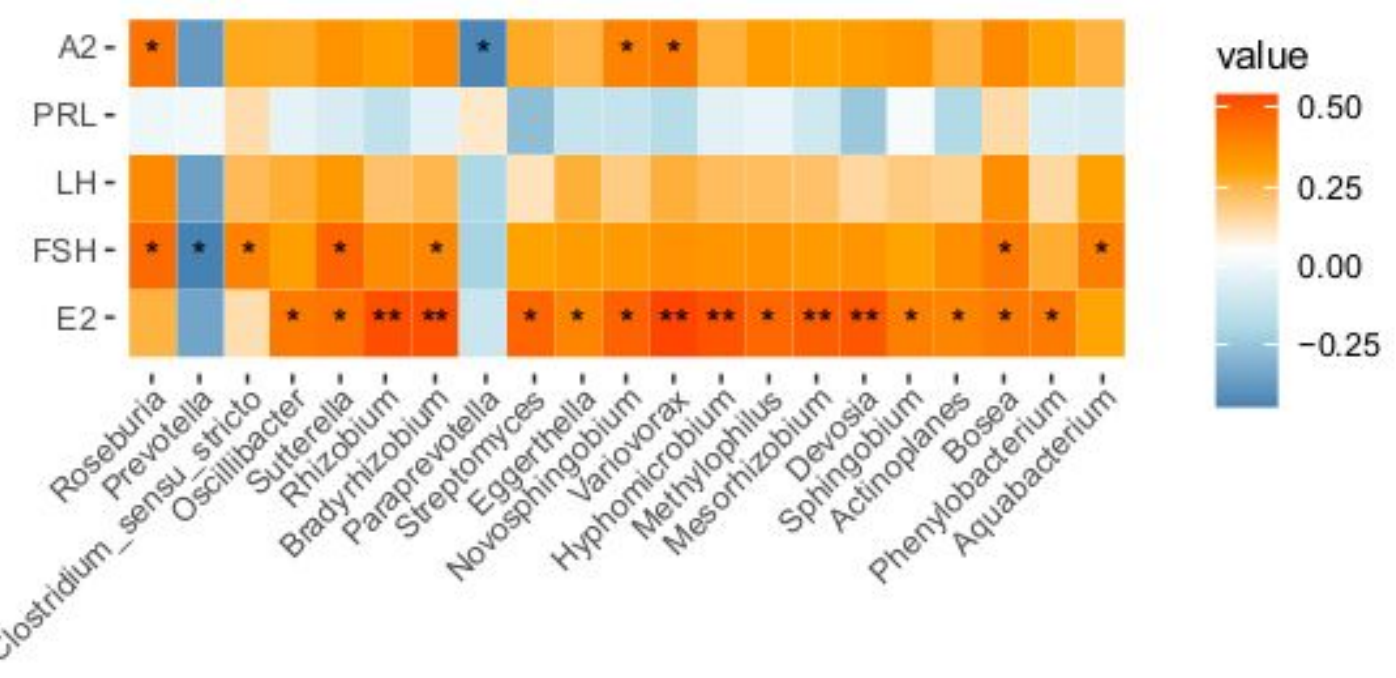

\section{Figure 3}

The correlation between GM and hormone levels in the ICPP group was analyzed. We found that the bacteria with higher abundance (Roseburia, Prevotella, and Fusicatenibacter) in the ICPP group were significantly associated with $\mathrm{FSH}, \mathrm{E} 2$, and A2.

\section{Supplementary Files}

This is a list of supplementary files associated with this preprint. Click to download.

- SupplementaryTable1.xls

- EthicsCommitteeStatement.pdf

- SupplementaryTable2.xlsx 